\title{
Underactuated Satellite Attitude Control with Two Parallel CMGs
}

\author{
Congying Han \\ Surrey Space Centre \\ University of Surrey \\ Guildford, GU2 7XH \\ UK \\ Email: C.Han@ surrey.ac.uk
}

\author{
Alexandre N. Pechev \\ Surrey Space Centre \\ University of Surrey \\ Guildford, GU2 7XH
}

$\mathrm{UK}$

Email: A.Pechev@surrey.ac.uk

\begin{abstract}
In this paper, we study the attitude stability and the disturbance attenuation properties for an underactuated spacecraft equipped with two parallel CMGs. We first present the actuator configuration and then compare this with typical fully actuated CMG-based configurations. The system model is then derived. Considering the pointing of an underactuated spacecraft, we derive a Lyapunov control law which is further modified to a dissipative controller to account for disturbances. Simulations are included to demonstrate the effectiveness of the proposed control law to attenuate perturbations and to render the underactuated attitude closed-loop system to a dissipative system.
\end{abstract}

\section{INTRODUCTION}

The Control Moment Gyro (CMG) offers high torque capability and high power and mass efficiency and therefore plays a key role in both satellite attitude stabilization and manoeuvring [1]. In addition to the existence of singular modes, the complicated structure of the $\mathrm{CMG}$ actuator demands hardware redundancies. For some applications with limited resources, redundances might not be a suitable option. Therefore a considerable amount of effort has gone in the past decades in the area of underactuated attitude control. Underactuated control is a scenario where fewer than three actuators are used to provide three axis control. Many results have been reported in cases of two pairs of thrusters and two reaction wheels [2][8]. The first application of underactuated attitude control was done for a system with two pairs of thrusters. In this case, only an asymmetric about the underactuated axis satellite can be controllable as the coupling terms in the underactuated axis can be used as a fictitious control input [5]-[7]. The attitude of the satellite can be globally stabilized by discontinuous time-invariant feedback or time varying feedback control [2][7]. Considering momentum exchange devices, most of the research effort was concentrated to systems where the tota angular momentum is zero, i.e. the attitude can be globally stabilized only if the initial angular velocity of the under actuated axis is zero and there is no disturbance about tha underactuated axis [9], [10]. From the results performed it orbit using two reaction wheels, the effect of a small non-zers momentum results in a rotation about the desired attitude [8]

In this paper, we study the underactuated control problem of a spacecraft equipped with two parallel CMGs. We seek for an efficient algorithm that is capable to deliver three axis attitude stabilization using two actuators under the influence of external disturbances. We make the assumption that there also exist other means for damping the total angular momentum every several orbits. In other words, the assumption is that although the satellite is perturbed by external disturbances, the total angular momentum is still kept at low values. We first present the actuator configuration and then compare this with typical fully actuated CMG-based configurations. The system model is then derived. In Section 3, the stability and disturbance attenuation properties are discussed. We demonstrate that there exists a control law which can not only stabilize the attitude system but also attenuate the disturbances leading to a dissipative system. Simulations are included to demonstrate the effectiveness in attenuating harmonic perturbations.

\section{UNDERACTUATED CMG ATTITUDE CONTROL}

\section{A. Attitude Control with CMGs}

The CMG produces torque by changing the direction of the angular momentum. With $\delta$ representing the gimbal angle and $h$ the angular momentum, the output torque $T$ delivered by the CMG can be written as $T=\dot{h}=J(\delta) \dot{\delta}$, where $J(\delta)$ is the Jacobian matrix. A difficulty associated with CMGs in attitude control loops is the existence of singular states. At a singularity, the CMG cannot generate a torque about a specific direction i.e., the rank of the Jacobian matrix is less than three. A special control algorithm, steering law, is used in conjunction with the attitude control loop to avoid or transit through the singularity. With the steering law in place, a typical attitude control loop with CMGs is presented in Fig 1. The steering law guarantees that the output torque of the CMGs $\dot{h}$ equals the desired torque $\dot{h}_{\text {des }}$.

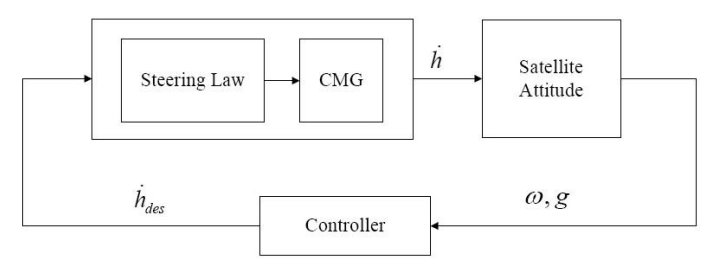

Fig. 1. Satellite attitude control loop with CMGs

In a system equipped with two CMGs, only two torque components are independent. For a configuration of two parallel CMGs, the torque about the third direction is zero. For a 
system with two CMGs, the rank of the Jacobian matrix can even drop to one, which implies that the torque produced by two actuators is along one of satellite's principle body axes. Under this situation, singularity is considered as the scenario where only one torque output becomes available or equivalently the rank of $J(\delta)$ is one. The problem of singular avoidance for an underactuated system is then transformed into a problem of how to manage the torque produced by two CMGs to be projected on the two axes.

For a system with two CMGs, in general only two torque outputs are independent and the third one is either zero or a combination of the other two. Using $\dot{h}_{1}, \dot{h}_{2}, \dot{h}_{3}$ to represent two of the CMG's torque components, $\dot{h}_{3}$ can be written as a function of the other two, i.e. $\dot{h}_{3}=f\left(\dot{h}_{1}, \dot{h}_{2}\right)$. For a given pair $\dot{h}_{1}, \dot{h}_{2}$, the linear function that depends on the physical arrangements of the actuators allows computing $\dot{h}_{3}$. The idea is depicted in Fig. 2. The task of the steering law is to deliver the identity $\dot{h}_{1}=\dot{h}_{1 \text { des }}, \dot{h}_{2}=\dot{h}_{2 \text { des }}$.

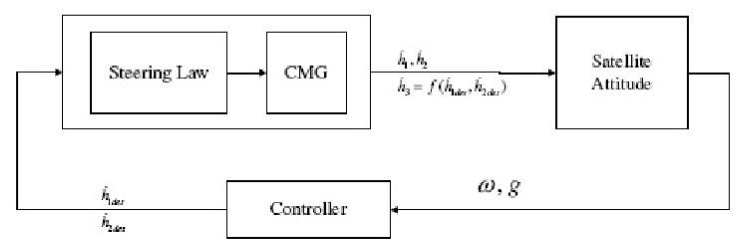

Fig. 2. Satellite attitude control loop with two CMGs

\section{B. Modeling of Underactuated Attitude Control System with Two $C M G s$}

1) Generic CMG pyramid configuration: Discarding the gimbal's inertia, the dynamic equation of a system with two CMGs can be written as

$$
I \dot{\omega}+\dot{h}+\omega \times(I \omega+h)=T_{e x t}
$$

where $I$ is the inertia matrix of the satellite, $\omega$ is the angular velocity, and $T_{\text {ext }}$ represents the external disturbances. Under the assumption that the total angular momentum is kept as zero, i.e. $I \omega+h \approx 0$ during several orbit periods, (1) reduces to

$$
I \dot{\omega}=-\dot{h}+T_{e x t}
$$

In a component form, (2) can be rewritten to

$$
\begin{aligned}
& I_{1} \dot{\omega}_{1}=-\dot{h}_{1}+T_{\text {ext } 1} \\
& I_{2} \dot{\omega}_{2}=-\dot{h}_{2}+T_{\text {ext } 2} \\
& I_{3} \dot{\omega}_{3}=-\dot{h}_{3}+T_{\text {ext } 3}
\end{aligned}
$$

So far this represents a generic case with three or more actuators. The underactuated configuration modifies the dynamic model to

$$
\begin{aligned}
& I_{1} \dot{\omega}_{1}=-\dot{h}_{1}+T_{e x t 1} \\
& I_{2} \dot{\omega}_{2}=-\dot{h}_{2}+T_{e x t 2} \\
& I_{3} \dot{\omega}_{3}=-f\left(\dot{h}_{1}, \dot{h}_{2}\right)+T_{e x t 3}
\end{aligned}
$$

Choosing Gibbs parameters $\left(g_{1}, g_{2}, g_{3}\right)$ to describe the attitude of the satellite and combining the kinematic equation with the dynamic equation, the complete mathematical model is obtained as

$$
\begin{aligned}
I_{1} \dot{\omega}_{1} & =-\dot{h}_{1}+T_{\text {ext } 1} \\
I_{2} \dot{\omega}_{2} & =-\dot{h}_{2}+T_{\text {ext } 2} \\
I_{3} \dot{\omega}_{3} & =-f\left(\dot{h}_{1}, \dot{h}_{2}\right)+T_{\text {ext } 3} \\
\dot{g}_{1} & =\frac{1+g_{1}^{2}}{2} \omega_{1}+\frac{g_{1} g_{2}-g_{3}}{2} \omega_{2}+\frac{g_{1} g_{3}+g_{2}}{2} \omega_{3} \\
\dot{g}_{2} & =\frac{g_{2} g_{1}+g_{3}}{2} \omega_{1}+\frac{1+g_{2}^{2}}{2} \omega_{2}+\frac{g_{2} g_{3}-g_{1}}{2} \omega_{3} \\
\dot{g}_{3} & =\frac{g_{1} g_{3}-g_{2}}{2} \omega_{1}+\frac{g_{1}+g_{2} g_{3}}{2} \omega_{2}+\frac{1+g_{3}^{2}}{2} \omega_{3}
\end{aligned}
$$

2) Configuration with two parallel CMGs: We now consider a case where two CMGs are installed in such a way that their gimbal axes are both aligned along the spacecraft's z-axis (Fig. 3). This results in torque components being projected only along $\mathrm{x}$ - and $\mathrm{y}$-axes.

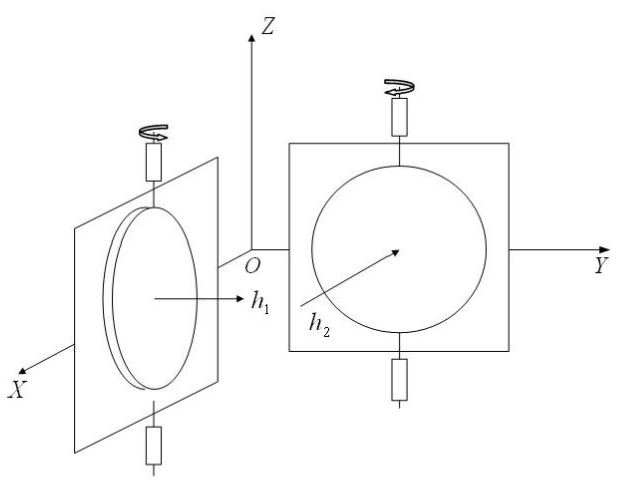

Fig. 3. Satellite attitude configuration with two parallel CMGs

Using (5), the complete mathematical model that describes the relationship between input torque and spacecraft attitude is given below

$$
\begin{aligned}
I_{1} \dot{\omega}_{1} & =-\dot{h}_{1}+T_{\text {ext } 1} \\
I_{1} \dot{\omega}_{2} & =-\dot{h}_{2}+T_{\text {ext } 2} \\
I_{1} \dot{\omega}_{3} & =T_{\text {ext } 3} \\
\dot{g}_{1} & =\frac{1+g_{1}^{2}}{2} \omega_{1}+\frac{g_{1} g_{2}-g_{3}}{2} \omega_{2}+\frac{g_{1} g_{3}+g_{2}}{2} \omega_{3} \\
\dot{g}_{2} & =\frac{g_{2} g_{1}+g_{3}}{2} \omega_{1}+\frac{1+g_{2}^{2}}{2} \omega_{2}+\frac{g_{2} g_{3}-g_{1}}{2} \omega_{3} \\
\dot{g}_{3} & =\frac{g_{1} g_{3}-g_{2}}{2} \omega_{1}+\frac{g_{1}+g_{2} g_{3}}{2} \omega_{2}+\frac{1+g_{3}^{2}}{2} \omega_{3}
\end{aligned}
$$




\section{STABILITY AND ROBUSTNESS ANALYSIS}

Defining a state vector constructed by two angular velocities and three attitude parameters, the controller design task is to obtain a feedback law that renders a closed loop system around a stable origin $(0,0,0,0,0)$. This has to be also guaranteed under the influence of external disturbances.

For the purpose of the controller design, we now construct a state-space representation of $(6)$

$$
\begin{aligned}
{\left[\begin{array}{c}
\dot{\omega}_{1} \\
\dot{\omega}_{2} \\
\dot{g}_{1} \\
\dot{g}_{2} \\
\dot{g}_{3}
\end{array}\right]=} & {\left[\begin{array}{c}
0 \\
\frac{1+g_{1}^{2}}{2} \omega_{1}+\frac{g_{1} g_{2}-g_{3}}{2} \omega_{2} \\
\frac{g_{2} g_{1}+g_{3}}{2} \omega_{1}+\frac{1+g_{2}^{2}}{2} \omega_{2} \\
\frac{g_{1} g_{3}-g_{2}}{2} \omega_{1}+\frac{g_{1}+g_{2} g_{3}}{2} \omega_{2}
\end{array}\right] } \\
& +\left[\begin{array}{cc}
-\frac{1}{I_{1}} & 0 \\
0 & -\frac{1}{I_{2}} \\
0 & 0 \\
0 & 0 \\
0 & 0
\end{array}\right]\left[\begin{array}{l}
\dot{h}_{1} \\
\dot{h}_{2}
\end{array}\right] \\
& +\left[\begin{array}{ccc}
\frac{1}{I_{1}} & 0 & 0 \\
0 & \frac{1}{I_{2}} & 0 \\
0 & 0 & \frac{g_{1} g_{3}+g_{2}}{2} \\
0 & 0 & \frac{g_{2} g_{3}-g_{1}}{2} \\
0 & 0 & \frac{1+g_{3}^{2}}{2}
\end{array}\right]\left[\begin{array}{l}
T_{\text {ext } 1} \\
\frac{T_{e x t 2}}{T_{e x t 3}}
\end{array}\right] \\
= & f(x)+B_{1} u+B_{2} w
\end{aligned}
$$

where $\overline{T_{\text {ext3 }}}=\int_{0}^{t} \frac{T_{\text {ext3 }}}{I_{3}} d t$

\section{A. Problem statement}

For the solution of the controller optimization task, a penalty function is defined as

$$
q=\left[\begin{array}{c}
c_{1}\left(\omega_{1}-\omega_{1 d}\right) \\
c_{2}\left(\omega_{2}-\omega_{2 d}\right) \\
c_{3} g_{1} \\
c_{4} g_{2} \\
c_{5} g_{3}
\end{array}\right]
$$

where $\omega_{1 d}, \omega_{2 d}$ are designed trajectories yet to be defined. The objective now is to find a state-feedback control law $u=$ $\left[\dot{h}_{1}, \dot{h}_{2}\right]^{T}$ and a positive definite and proper function $V$, such that for $\gamma>0$, along the trajectories of the close-loop system, one has [11]

$$
H J I(u, V)=V_{x}(f(x)+B u)+V_{x} \frac{B_{2} B_{2}^{T}}{4 \gamma^{2}} V_{x}^{T}+q^{T} q<0
$$

This guarantees that the energy of the penalty function is less than or equal to the energy of the disturbance input.

\section{B. Main result}

\section{Proposition}

Consider (7) and the penalty function (8), there exists a pair $(u, V)$ such that $H J I(u, V)<0$.

\section{Proof}

$$
\begin{aligned}
& \text { Let } u=\left[\begin{array}{c}
\dot{h}_{1} \\
\dot{h}_{2}
\end{array}\right]=\left[\begin{array}{l}
I_{1}\left[k_{1}\left(\omega_{1}-\omega_{1 d}\right)-\dot{\omega}_{1 d}\right] \\
I_{2}\left[k_{2}\left(\omega_{2}-\omega_{2 d}\right)-\dot{\omega}_{2 d}\right]
\end{array}\right] \\
& \qquad V=\frac{\left(\omega_{1}-\omega_{1 d}\right)^{2}}{2}+\frac{\left(\omega_{2}-\omega_{2 d}\right)^{2}}{2}+\frac{g_{1}^{2}}{2}+\frac{g_{2}^{2}}{2}+\frac{g_{3}^{2}}{2}
\end{aligned}
$$

with

$$
\begin{aligned}
\omega_{1 d} & =\frac{2}{1+g_{1}^{2}+g_{2}^{2}+g_{3}^{2}}\left[\left(1+g_{2}^{2}\right) \nu_{1 d}+\left(-g_{1} g_{2}+g_{3}\right) \nu_{2 d}\right] \\
\omega_{2 d} & =\frac{2}{1+g_{1}^{2}+g_{2}^{2}+g_{3}^{2}}\left[\left(1+g_{1}^{2}\right) \nu_{2 d}+\left(-g_{1} g_{2}-g_{3}\right) \nu_{1 d}\right] \\
\nu_{1 d} & =-k g_{1}+\mu \frac{g_{2} g_{3}}{g_{1}^{2}+g_{2}^{2}+0.001} \\
\nu_{2 d} & =-k g_{2}-\mu \frac{g_{1} g_{3}}{g_{1}^{2}+g_{2}^{2}+0.001}
\end{aligned}
$$

and $k>0, \mu>0, k_{1}>0, k_{2}>0$ [5], [8]. At that point we conclude that any positive gain parameters in the closed-loop above will render to a stable closed-loop system.

Furthermore, to analyze the disturbance attenuation properties, we use the Hamiltonian-Jacobian-Isaac inequality to pose some constraints on the choice of the controller gains. After simple but lengthy calculations this yields

$$
\begin{aligned}
H J I(u, V)= & -\left(k_{1}-\frac{1}{4 \gamma^{2} I_{1}}-c_{1}^{2}\right)\left(\omega_{1}-\omega_{1 d}\right)^{2} \\
& -\left(k_{2}-\frac{1}{4 \gamma^{2} I_{2}}-c_{2}^{2}\right)\left(\omega_{2}-\omega_{2 d}\right)^{2} \\
& -k g_{1}^{2}-k g_{2}^{2}-\mu g_{3}^{3}+c_{3}^{2} g_{1}^{2}+c_{4}^{2} g_{2}^{2}+c_{5}^{2} g_{3}^{2} \\
& +\frac{1}{4 \gamma^{2}} g_{3}^{2}\left(\frac{g_{1}^{2}}{2}+\frac{g_{2}^{2}}{2}+\frac{g_{3}^{2}}{2}+\frac{1}{2}\right)^{2} \\
= & -\left(k_{1}-\frac{1}{4 \gamma^{2} I_{1}}-c_{1}^{2}\right)\left(\omega_{1}-\omega_{1 d}\right)^{2} \\
& -\left(k_{2}-\frac{1}{4 \gamma^{2} I_{2}}-c_{2}^{2}\right)\left(\omega_{2}-\omega_{2 d}\right)^{2} \\
& -\left(k-c_{3}^{2}\right) g_{1}^{2}-\left(k-c_{4}^{2}\right) g_{2}^{2} \\
& -\left[\mu-\frac{1}{4 \gamma^{2}}\left(\frac{g_{1}^{2}}{2}+\frac{g_{2}^{2}}{2}+\frac{g_{3}^{2}}{2}+\frac{1}{2}\right)^{2}-c_{5}^{2}\right] g_{3}^{2}
\end{aligned}
$$

This representation suggests that there exists a choice of the gains such that $H J I$ becomes negative for state trajectories that belong to a compact set such that $\left(\omega_{1 d}, \omega_{2 d}, 0,0,0\right)$ belongs to it. Therefore, selecting $k_{1}>\frac{1}{4 \gamma^{2} I_{1}}+c_{1}^{2}, k_{2}>$ $\frac{1}{4 \gamma^{2} I_{2}}+c_{2}^{2}, k>\max \left(c_{3}^{2}, c_{4}^{2}\right), \mu>\frac{1}{4 \gamma^{2}}\left(\frac{g_{1}^{2}}{2}+\frac{g_{2}^{2}}{2}+\frac{g_{3}^{2}}{2}+\frac{1}{2}\right)^{2}+c_{5}^{2}$ results in $H J I(u, V)<0$.

\section{Discussion}

1) System Stability: Firstly, we set the disturbances to zero and consider only system stability. Substituting $u$ into the system's model transforms (7) to 


$$
\left[\begin{array}{c}
\dot{\omega}_{1} \\
\dot{\omega}_{2} \\
\dot{g}_{1} \\
\dot{g}_{2} \\
\dot{g}_{3}
\end{array}\right]=\left[\begin{array}{c}
-k_{1}\left(\omega_{1}-\omega_{1 d}\right)+\dot{\omega}_{1 d} \\
-k_{2}\left(\omega_{2}-\omega_{2 d}\right)+\dot{\omega}_{2 d} \\
-k g_{1}+\mu \frac{g_{2} g_{3}}{g_{1}^{2}+g_{2}^{2}} \\
-k g_{2}-\mu \frac{g_{1} g_{3}}{g_{1}^{2}+g_{2}^{2}} \\
-\mu g_{3}
\end{array}\right]
$$

Equation (11) shows that if the attitude parameters $g_{1}$ and $g_{2}$ can be driven along a desired trajectory by two angular velocities, the third parameter $g_{3}$ can be also controlled. This results in a three axis control with two actuators. The terms $\left(-k g_{1},-k g_{2}\right)$ in the expression $\nu_{1 d}, \nu_{2 d}$ stabilize $\left(g_{1}, g_{2}\right)$, whereas the coupling terms $\left(\mu \frac{g_{2} g_{3}}{g_{1}^{2}+g_{2}^{2}}, \mu \frac{g_{1} g_{3}}{g_{1}^{2}+g_{2}^{2}}\right)$ contribute to the stability on the underactuated $\mathrm{z}$-axis. Large values for $k$ result in fast convergence for $\left(g_{1}, g_{2}\right) . \mu$, on another hand, determines the coupling ration and hence the convergence rate for the underactuated axis.

2) Disturbances attenuation: The proposition here is that there exist a torque disturbance on the spacecraft such that all three axes are perturbed. Using the developments from the previous section, we have demonstrated that three axis control and disturbance attenuation are possible. The control law can bring the system into a stable equilibrium point against worstcase input. It can be shown that the attitude of the satellite will convergent to the region $g_{1}^{2}+g_{2}^{2}+g_{3}^{2}<4 \gamma \sqrt{\mu-c_{5}^{2}}-1$ when $k_{1}>\frac{1}{4 \gamma^{2} I_{1}}+c_{1}^{2}, k_{2}>\frac{1}{4 \gamma^{2} I_{2}}+c_{2}^{2}, k>\max \left(c_{3}^{2}, c_{4}^{2}\right)$. It is evident from this expression that the size of the region is controlled by $\mu$.

\section{SIMULATION}

We now present some simulation results. We recall to the fact that the following inequities need to be satisfied $k_{1}>$ $\frac{1}{4 \gamma^{2}}+c_{1}^{2}, k_{2}>\frac{1}{4 \gamma^{2}}+c_{2}^{2}, k>\max \left(c_{3}^{2}, c_{4}^{2}\right), \mu>\frac{1}{4 \gamma^{2}}\left(\frac{g_{1}^{2}}{2}+\frac{g_{2}^{2}}{2}+\right.$ $\left.\frac{9_{3}^{2}}{2}+\frac{1}{2}\right)^{2}+c_{5}^{2}$ in order to make all $H J I(u, V)<0$ for all $\omega_{1} \neq \omega_{1 d}, \omega_{1} \neq \omega_{2 d}, g_{1} \neq 0, g_{2} \neq 0$ and $g_{3} \neq 0$. The design parameters are set to $c_{1}=c_{2}=1, c_{3}=c_{4}=c_{5}=2$. For the purpose of the analysis, $k_{1}, k_{2}$ and $k$ are grouped together and $\mu$ is separated. This allows us to study performance by satisfying one or another group of parameters. We first set $k_{1}=10, k_{2}=10, k=\mu=5$ and then to $k_{1}=0.8$, $k_{2}=0.8, k=\mu=1$. In the first case both the stability and the disturbance attenuation properties are satisfied. For the second set we can only guarantee the stability of the control loop. In both cases, the disturbance on each axis is set to a sinusoidal signal of amplitude 0.01 and frequency one. Fig. 4 and 5 display the attitude history from initial conditions set to $(2,1,1)$. For the analysis of the disturbance attenuation properties, we also display the value of the $H J I$ function for different gains.

The simulation results show that both combinations in the controller gains give a sable closed-loop system and a three axis stabilization using only two torque inputs. Figs. 4 and 5 , however, also show that by choosing the gains such that the $H J I$ is satisfied, better disturbance attenuation can be achieved. It has to be noted, however, that although higher controller gains might improve on the precision, care has to be taken not to saturate the actuators. We here argue that due to

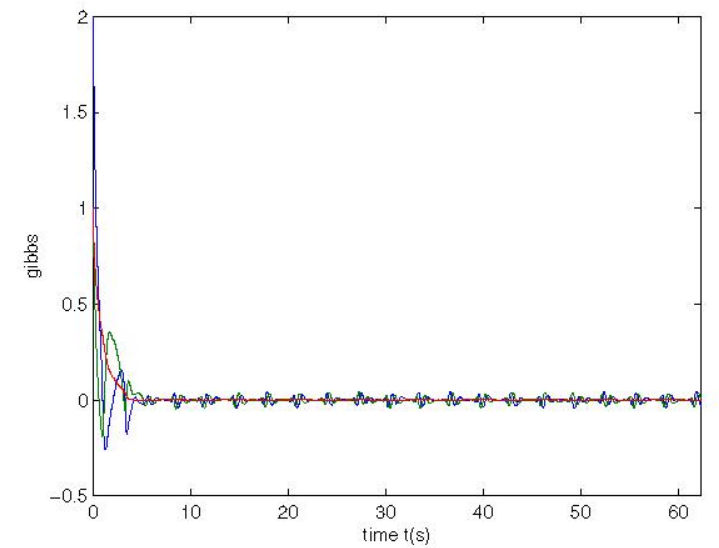

(a) Attitude

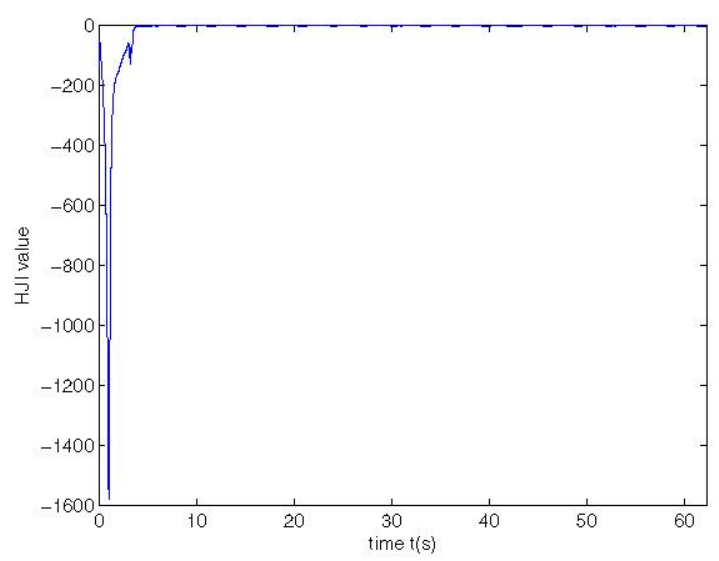

(b) HJI value

Fig. 4. Attitude and $H J I$ value histories from initial condition $(2,1,1)$ for $k_{1}=10, k_{2}=10, k=\mu=5$

the high torque capacity of the CMG actuator, better precision might be expected in an underactuated scenario.

\section{CONCLUSIONS}

Underactuated attitude control of satellites equipped with two parallel CMGs is investigated. A control law is derived and a set of controller gains that guarantee stability and disturbance attenuation properties through a dissipation analysis. The controller manages to deliver three axis attitude control with only two torque inputs under the influence of disturbances. A compact set has been derived where all states would reside at the end of the transient and this set has been connected with the controller gains. We also note that $\mu$ is the only parameter that depends on the state vector. In our current research work we investigate the suitability for adapting it, for example by taking $\mu=\frac{1}{4 \gamma^{2}}\left(\frac{g_{1}^{2}}{2}+\frac{g_{2}^{2}}{2}+\frac{g_{3}^{2}}{2}+\frac{1}{2}\right)^{2}+c_{5}^{2}+\epsilon, \epsilon>0$, $H J I$ (9) can be rendered to be negative for all $\omega_{1} \neq \omega_{1 d}$, $\omega_{1} \neq \omega_{2 d}, g_{1} \neq 0, g_{2} \neq 0$ and $g_{3} \neq 0$. The authors are also working on problems where the control moment gyroscopes 


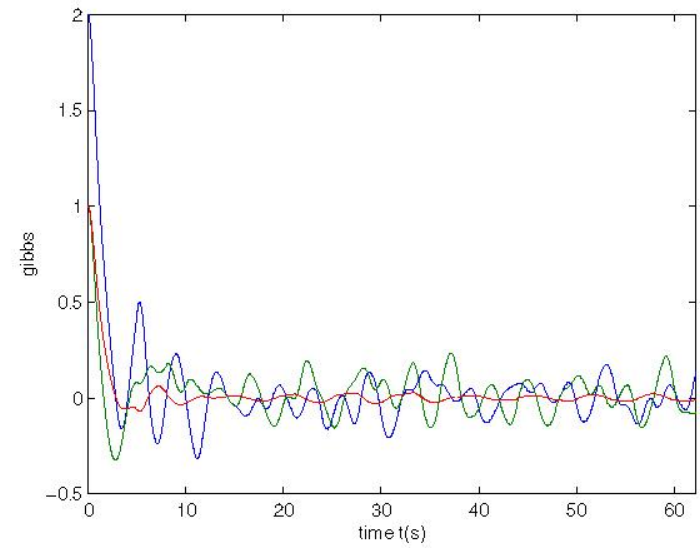

(a) Attitude

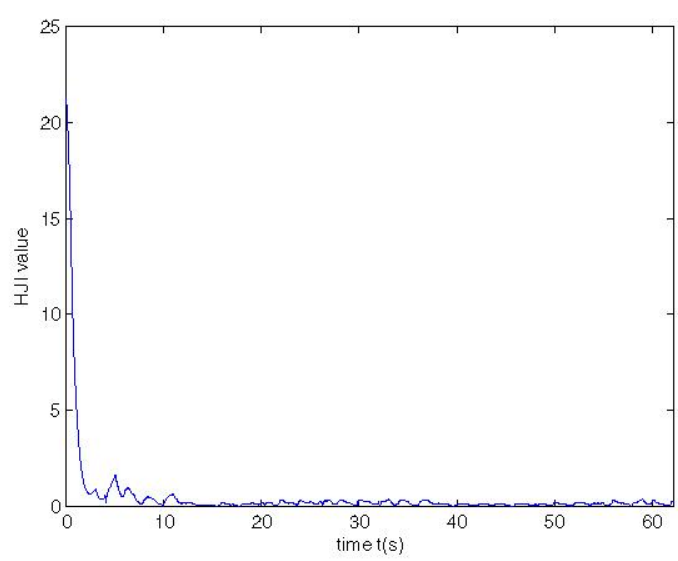

(b) $H J I$ value

Fig. 5. Attitude and $H J I$ value histories from initial condition $(2,1,1)$ for $k_{1}=0.8, k_{2}=0.8, k=\mu=1$

are configured in different arrangements. An experimental test rig for validation of underactuated control algorithms is also under development.

\section{ACKNOWLEDGMENT}

The authors would like to acknowledge the support of the Surrey Space Centre, University of Surrey.

\section{REFERENCES}

[1] Margulies, G. and Aubrun, J. N."'Geometric theory of single-gimbal control moment gyro systems," The Joumal of the Astronautical Sciences, Vol.26, pp.159-191, 1978.

[2] P.Morin, "Time varying exponential stabilization of the attitude of a rigid spacecraft with two control torques", IEEE Transactions on automatic control, Vol.42, No.4, 1997

[3] G.C.Walsh, "Orientation control of the dynamic satellite", Proceedings of the 1994 American Control Conference,pp.138-142, 1994

[4] O.J.Sordalen, "Attitude stabilization with nonholonomic constraints", Proceedings of the 31 st IEEE conference on Decision and Control, Vol.2, pp. $1610-1611,1992$.
[5] P.Tsiotras, "Control of spacecraft subject to actuator failures: State of the art and open problems", Richard H.Battin Astrodynaics Conference, American Astronotical society, AAS 00-264, 2000.

[6] S.Kim, "Sliding mode stabilizing control law of underactuated spacecraft", AIAA Guidance, Navigation and control Conference and Exhibit, Denver, CO, 14-17 August 2000.

[7] K.M.Fauske, "Attitude stabilization of an underactuated rigid spacecraft", SIVING thesis, Norwegian university of technology and science, 2003.

[8] N.M.Horri, "Attitude control of underactuatede small satellites", Doctor thesis, University of Surrey, 2004.

[9] HKrishman, "Attitude stabilization of a rigid spacecraft using two momentum wheel actuators", AIAA Joumal of Guidance, Control and Dynamics, 1994.

[10] K.Yamada, "Feedback attitude control of a spacecraft by two reaction wheels", 2Ist International Symposium on Space Technology and Science, Omiya, Japan, 1998.

[11] A.Astolfi and A.Rapaport, "Robust stabilization of the angular velocity of a rigid body" Systems \& Control Letters, Vol.34, No.5, pp.257-264, 1998. 